\title{
MATHEMATiCAL Modelling OF EPIDEMiology in PRESENCE OF VACCINATION AND DELAY
}

\author{
Uttam Ghosh $^{1}$, Sourav Chowdhury ${ }^{2}$ and Dilip Kumar Khan ${ }^{3}$ \\ ${ }^{1}$ Department of Mathematics, Nabadwip Vidyasagar College, Nadia, West \\ Bengal, \\ uttam_math@yahoo.co.in \\ ${ }^{2}$ Department of Applied Science \& Humanities, Global Institute of Management \\ and Technology, Krishnagar, Nadia. \\ souravformanuegmail. com \\ ${ }^{3}$ Department of Environmental Science, University of Kalyani, Kalyani, Nadia, \\ West Bengal
}

\begin{abstract}
The Mathematical modeling of infectious disease is currently a major research topic in the public health domain. In some cases the infected individuals may not be infectious at the time of infection. To become infectious, the infected individuals take some times which is known as latent period or delay. Here the two SIR models are taken into consideration for present analysis where the newly entered individuals have been vaccinated with a specific rate. The analysis of these models show that if vaccination is administered to the newly entering individuals then the system will be asymptotically stable in both cases i.e. with delay and without delay.
\end{abstract}

\section{KEYWORDS}

Epidemic modeling, Infectious disease, susceptible, asymptotically stable.

\section{INTRODUCTION}

The use of Mathematical model for different epidemic studies is done by different authors. The non-linear incidence rate of saturated mass action is used by Liu et al [6], Ruan and Wang [2]. The easy way to control the disease is the vaccination of the infected or susceptible in different stages. The SIS model with vaccination, standard incidence with no disease induced death was investigated by Kribs-Zeleta and Velasco-Hernodez [5]. SIRS model with vaccination, standard incidence and no disease induced death was investigated by Arino et al [3]. Different vaccination models with standard incidence rate and considering disease induced death was investigated by different authors (Braur [4], Hui and Zhu [8], Li et al [7]). Those models were studied taking vaccination to the susceptible individuals and without considering the delay of the infected individuals. Vaccination to the newly appointed individuals and considering the delay of the infected individuals was studied by different authors. Kaddar [1] investigated the stability of delayed SIR epidemic model considering saturated incidence rate. Cai and Li [10] investigated SEIV epidemic model with nonlinear incidence rate and they concluded that the necessary and important aspect for public health-management is to control an epidemic by increasing the duration of loss of immunity induced by vaccination. Here the model is considered to investigate

Rupak Bhattacharyya et al. (Eds) : ACER 2013,

pp. 91-98, 2013. (C) CS \& IT-CSCP 2013

DOI : $10.5121 /$ csit.2013.3209 
the epidemic model in presence of vaccination to the newly entering individuals and considering the delay $(\tau)$ for the infected individuals to become infectious and the case $\tau=0$ is also discussed, the similar problem without-delay is also discussed by Mena-Lorca and Hethcote [9] without considering the vaccination processes.

\section{MATHEMATICAL FORMULATION}

Let $S(t)$ be the number of susceptible and $I(t)$ be the number of infected and $R(t)$ be the number of recovered individual such that $\mathrm{N}(\mathrm{t})=\mathrm{S}(\mathrm{t})+\mathrm{I}(\mathrm{t})+\mathrm{R}(\mathrm{t})$. Since the spreading of disease can be controlled by the vaccination to the individuals. Here the SIR model is considered in presence of vaccination to the new entrants (i.e. at the time of birth or at the time of immigration). Let $\mathbf{b}$ be the number of newly appointed population and $\mathbf{p}$ be the percentage of population with vaccination for the newly entering population then (1-p)b is the unvaccinated but susceptible individuals. Then the differential equation of the model (Model-I) will be

$$
\left.\begin{array}{l}
\frac{d S}{d t}=b(1-p)-\beta I S-d S+\gamma R \\
\frac{d I}{d t}=\beta I S-\gamma-d I \\
\frac{d R}{d t}=\gamma-(d+\gamma) R
\end{array}\right\}
$$

Where $\mathrm{d}$ is the death rate and $\gamma$ be the recovery rate. But in some disease the infected individuals are not infectious at the time of infection. Let $\tau$ be the latent period or period of incubation of the infection and assume that the susceptible individuals infected at time $\mathrm{t}-\tau$ and become infectious at time t. Then the differential equation of the model considering delay (Model-II) will be

$$
\left.\begin{array}{l}
\frac{d S}{d t}=b(1-p)-\beta I S-d S+\gamma R \\
\frac{d I}{d t}=e^{-d \tau} \beta I(t-\tau) S(t-\tau)-\gamma I-d I \\
\frac{d R}{d t}=\gamma I-(d+\gamma) R
\end{array}\right\} \ldots \ldots \ldots \ldots \ldots(
$$

\subsection{Stability analysis of Model-I}

For Model-I, the equilibrium can be found by setting $\frac{d S}{d t}=\frac{d R}{d t}=\frac{d I}{d t}=0$, with points $\mathrm{A}_{10}\left(\mathrm{~S}_{0}, \mathrm{R}_{0}, \mathrm{I}_{0}\right)$ and $\mathrm{A}_{11}\left(\mathrm{~S}_{1}, \mathrm{I}_{1}, \mathrm{R}_{1}\right)$ where $\mathrm{R}_{0}=0, \mathrm{I}_{0}=0, S_{1}=\frac{\gamma+d}{\beta}$,

$$
I_{1}=\frac{\left(R_{01}-1\right)(d+\gamma)}{d+2 \gamma}, R_{1}=\frac{\gamma}{\gamma+d} I_{1} \cdot R_{01}=\frac{b_{1} \beta}{d(d+\gamma)}, \quad S_{0}=b_{1} / d, b_{1}=b(1-p) . \quad \mathrm{A}_{10} \quad \text { is }
$$

disease free equilibrium point, $\mathrm{A}_{11}$ is other equilibrium point, $\mathrm{A}_{11}$ will exists when $b_{1} \beta-d(d+\gamma)>0$, therefore the disease free equilibrium point exists for all $\mathrm{R}_{01}$ and the endemic equilibrium point will exists when $\mathrm{R}_{01}>1$.

The corresponding Jacobian for (1) is 


$$
J=\left(\begin{array}{ccc}
-(d+\beta I) & -\beta S & \gamma \\
\beta I & \beta S-(d+\gamma) & 0 \\
0 & \gamma & -(d+\gamma)
\end{array}\right)
$$

The eigen values of the Jacobean for the disease free equilibrium point $A_{10}\left(d_{1} / b, 0,0\right)$ are $\lambda=-d,-(\gamma+d)$ and $\beta S_{0}-(\gamma+d)$. Since two of the three eigen values are always negative and other will be negative if $\mathrm{R}_{01}<1$ and the solution in the neighborhood of disease free equilibrium will be asymptotically stable. If $R_{01}>1$ the one of the root will be positive and the equilibrium point will be saddle point and the corresponding solution will be unstable in nature. Therefore, the disease free equilibrium point will be locally asymptotically stable for $\mathrm{R}_{01}<1$.To cheek the global stability by considering for $\mathrm{R}_{01}<1$.

Again summing up the equations in (1) we get

$$
\begin{aligned}
& \frac{d N}{d t}=b_{1}-d N \\
& \text { or, } N=\frac{b_{1}}{d}+C e^{-d t} \rightarrow \frac{b_{1}}{d} \text { as } t \rightarrow \infty
\end{aligned}
$$

Where $\mathbf{c}$ is constant.

Therefore, the region $\mathrm{D}=\left\{(\mathrm{S}, \mathrm{I}, \mathrm{R}): \mathrm{S}+\mathrm{I}+\mathrm{R} \leq \frac{b_{1}}{d}\right\}$ is positively in variant set for $(1)$.

Considering the Liapunov function $\mathrm{L}=\mathrm{I}$

$$
\begin{aligned}
\frac{d L}{d t}=\frac{d I}{d t}= & (\beta S-(\gamma+d)) I \\
& \leq\left(\beta S_{0}-(\gamma+d)\right) I \\
& =(d+\gamma)\left(R_{01}-1\right) I \leq 0 \text { for } R_{01}<1
\end{aligned}
$$

Then $\frac{d I}{d t}=0$ only when $\mathrm{I}=0$, therefore the only positively invariant subset of the plane $\mathrm{I}=0$ is the point $\mathrm{A}_{10}$. Thus it follows from the Lasalle-Liapunov theory (Hale [12] Ma and Li [11]) the disease free equilibrium point is globally stable.

Characteristic equation for the endemic equilibrium point $\left(S_{1}, I_{1}, R_{1}\right)$ is

$$
\left|\begin{array}{ccc}
-\left(d+\beta I_{1}\right)-\lambda & -\beta S_{1} & \gamma \\
\beta I_{1} & \beta S_{1}-(d+\gamma)-\lambda & 0 \\
0 & \gamma & -(d+\gamma)-\lambda
\end{array}\right|=0
$$

Simplifying the above 


$$
\begin{aligned}
& \lambda^{3}+C_{1} \lambda^{2}+C_{2} \lambda+C_{3}=0 \\
& \text { Where } C_{1}=2 d+\gamma+\beta I_{1} \\
& C_{2}=(\gamma+d)\left\{\left(d+2 \beta I_{1}\right)\right\} \\
& C_{3}=\beta I_{1}\left(d^{2}+2 d \gamma\right) \\
& C_{1} C_{2}-C_{3}=d(\gamma+d)(\gamma+2 d)+\beta I_{1}\left\{2 \beta I_{1}(\gamma+d)+4 d^{2}+5 d \gamma+2 \gamma^{2}\right\}
\end{aligned}
$$

Since all the coefficients will be positive only when $\mathrm{R}_{01}>1$, then $C_{1} C_{2}-C_{3}>0$. Therefore, all roots of the equation (3) have negative real part (Routh-Horwtz criteria).

\subsection{Stability analysis for Model-II}

With equilibrium points $\left(\mathrm{S}_{0}, \mathrm{R}_{0}, \mathrm{I}_{0}\right)=\left(\mathrm{b}_{1} / \mathrm{d}, 0,0\right)$ which is disease free equilibrium point and $\left(S_{1}^{*}, I_{1}^{*}, R_{1}^{*}\right) \quad$ is the endemic equilibrium point. Where $S_{1}^{*}=\frac{S_{0}}{I_{0}}, I_{1}^{*}=\frac{b_{1}(d+\gamma)\left(R_{0}-1\right)}{\gamma^{2} R_{0}\left(R_{2}-1\right)}$ and $R_{1}^{*}=\frac{\gamma}{\gamma+d} I_{1}^{*}$ and $\quad R_{2}=\frac{\beta S_{0}(d+\gamma)}{\gamma^{2} R_{0}} \quad$ and $\quad$ this equilibrium point exists when $\mathrm{R}_{2}>1$ and $\mathrm{R}_{0}>1$.

Linearzing the system (II) about the point $\left(\mathrm{S}_{0}, 0,0\right)$, by setting $S=\mathrm{S}^{\prime}+\mathrm{S}_{0}, \mathrm{I}=\mathrm{I}^{\prime}$ and $\mathrm{R}=\mathrm{R}^{\prime}$, rewriting the equations omitting the dot-sign we get

$$
\left.\begin{array}{l}
\frac{d S}{d t}=-\beta I S_{0}-d S+\gamma R \\
\frac{d I}{d t}=e^{-d \tau} \beta I(t-\tau) S_{0}(t-\tau)-(\gamma+d) I \\
\frac{d R}{d t}=\gamma-(d+\gamma) R
\end{array}\right\} \ldots \ldots \ldots \ldots . . .(3)
$$

The characteristic roots of the corresponding linearzing system are $\lambda=-d,-(d+\gamma)$ and other root satisfies the equation $\lambda=e^{-(d+\lambda) \tau}-(d+\gamma)$.

Since the two roots are negative and the third root satisfies the transcendental equation (4).

For $\tau=0$ the above roots all becomes negative, therefore, all the roots of the characteristics equation have negative real part, therefore, the system will be asymptotically stable in the neighborhood of the disease free equilibrium point for $\tau=0$, By Rouche's theorem it follows that if the instability occurs for a particular value of $\tau$, a characteristic root of the equation (4) must intersect the imaginary axis. Suppose (4) has a purely imaginary root $i \omega, \omega>0$ then from (4) the following relation will be obtained

$$
i \omega=\beta e^{-d \tau} S_{0}(\operatorname{Cos} \lambda \omega-i \operatorname{Sin} \lambda \omega)-(\gamma+d) .
$$

Comparing real and imaginary parts in both sides we get 


$$
\left.\begin{array}{l}
\omega=\beta e^{-d \tau} S_{0} \operatorname{Cos} \lambda \omega \\
\gamma+d=\beta e^{-d \tau} S_{0} \operatorname{Sin} \lambda \omega
\end{array}\right\}
$$

Squaring and adding the above two we get

$$
\begin{aligned}
& \omega^{2}+(\gamma+d)^{2}=\beta^{2} e^{-2 d \tau} S_{0}^{2} \\
& \omega^{2}=(\gamma+d)^{2}\left\{R_{0}^{2}-1\right\}
\end{aligned}
$$

Since $\mathrm{R}_{0}<1$, the equation (5) has no positive root. Therefore, the disease free equilibrium $\left(\mathrm{S}_{0}, 0\right.$, $0)$ is locally asymptotically stable. If $R_{0}>1$, then the disease free equilibrium $\left(S_{0}, 0,0\right)$ is unstable for $\tau=0$. By Kuang theorem the equilibrium point $\left(S_{0}, 0,0\right)$ is unstable for all $\tau \geq 0$.

Again linearzing the system (II) about the point $\left(S_{1}^{*}, I_{1}^{*}, R_{1}^{*}\right)$ put

$$
S=S^{\prime}+S_{1}^{*}, I=I^{\prime}+I_{1}^{*}, R=R^{\prime}+R_{1}^{*} \text {, rewriting the equations omitting the dot-sign we get }
$$

$$
\left.\begin{array}{l}
\frac{d S}{d t}=-\beta I S_{1}^{*}-\left(d+\beta I_{1}^{*}\right) S+\gamma R \\
\frac{d I}{d t}=e^{-d \tau} \beta\left\{I(t-\tau) S_{1}^{*}+I_{1}^{*} S(t-\tau)\right\}-(\gamma+d) I \\
\frac{d R}{d t}=\gamma-(d+\gamma) R
\end{array}\right\}
$$

Then the characteristics equation becomes

$$
\begin{aligned}
& \begin{aligned}
& \lambda^{3}+A \lambda^{2}+B \lambda+C+e^{-(\lambda+d) \tau}\left\{-\lambda^{2} \beta S_{1}^{*}+\lambda \beta S_{1}^{*}(2 d+\gamma)-\left(d(d+\gamma)^{2}+\beta I_{1}^{*} \gamma^{2}\right)\right\}=0 . . . \\
& \text { where } A=3 d+2 \gamma+\beta I_{1}^{*} \\
& \qquad B=(d+\gamma)\left(3 d+\gamma+2 \beta I_{1}^{*}\right) \\
& C=(d+\gamma)^{2}\left(d+\beta I_{1}^{*}\right)
\end{aligned}
\end{aligned}
$$

For $\tau=0$ equation (6) becomes

$$
\begin{aligned}
& \lambda^{3}+C_{1} \lambda^{2}+C_{2} \lambda+C_{3}=0 \ldots \ldots \ldots \ldots \ldots \ldots \ldots \ldots . . .(7) \\
& \begin{aligned}
\text { Where } C_{1}=2 d+\gamma+ & \left.\beta I_{1}^{*}, C_{2}=(d+\gamma)\left(3 d+\gamma+2 \beta I_{1}^{*}\right)+(2 d+\gamma) \beta S_{1}^{*}, \quad C_{3}=\left(2 d \gamma+d^{2}\right) \beta I_{1}^{*}\right) \\
C_{1} C_{2}-C_{3}= & (2 d+\gamma)\left\{(d+\gamma)\left(2 \beta I_{1}^{*}+3 d+\gamma\right)+(2 d+\gamma) \beta S_{1}^{* *}\right\} \\
& +2(d+\gamma) \beta^{2} I_{1}^{* 2}+2 \beta I_{1}^{*}\left(4 d^{2}+5 \gamma d+2 \gamma^{2}\right)
\end{aligned}
\end{aligned}
$$

and then values of $C_{1}, C_{2}, C_{3}$ and $C_{1} \cdot C_{2}-C_{3}$ all positive and therefore by Routh-Harwtz criteria the roots of equation (7) have all negative real part. Therefore for $\tau=0$ the system is asymptotically stable. Arguing as previous for the disease free equilibrium point and Rouche's theorem the purely imaginary root of the equation (6) can be taken as $i \omega, \omega>0$, then separating real and imaginary part in equation (6) after putting $\lambda=i \omega, \omega>0$ 


$$
\begin{aligned}
& -\left(p \omega^{2}-r\right) \operatorname{Cos} \omega \tau+q \operatorname{\omega S} \operatorname{Sin} \omega \tau=C-A \omega^{2} \\
& q \omega \operatorname{Cos} \omega \tau+\left(p \omega^{2}-r\right) \operatorname{Sin} \omega \tau=B \omega-\omega^{3} \\
& \text { Where } p=d+\gamma, q=(d+\gamma)(2 d+\gamma), r=\left(d(d+\gamma)^{2}+\beta I_{1}^{*}\right) e^{-d \tau}
\end{aligned}
$$

Squaring and adding the above we get

$$
\begin{aligned}
& \left(p \omega^{2}-r\right)^{2}+q^{2} \omega^{2}=\left(C-A \omega^{2}\right)^{2}+\left(B \omega-\omega^{3}\right)^{2} \\
& \omega^{6}+\omega^{4}\left(A^{2}-p^{2}-2 B\right)+\omega^{2}\left(B^{2}-2 A C+2 p r-q^{2}\right)+C^{2}-r^{2}=0 \\
& A^{2}-p^{2}-2 B=\beta^{2} I_{1}^{* 2}+2 d \beta I_{1}^{*}+2 d^{2}+2 d \gamma+\gamma^{2}>0 \\
& B^{2}-2 A C+2 p r-q^{2}=\left\{2 \beta^{2} I_{1}^{* 2}+4 d \beta I_{1}^{*}+5 d^{2}+2 d \gamma\right\}>0 \\
& C^{2}-r^{2}>2 d \beta I_{1}^{*}(d+\gamma)^{2}\left\{(d+\gamma)^{2}-\gamma^{2}\right\}+\beta^{2} I_{1}^{* 2}\left\{(d+\gamma)^{4}-\gamma^{4}\right\}>0
\end{aligned}
$$

The above equation is a cubic in $\omega^{2}$ having all positive coefficient and therefore no root of the equation (8) is positive for $R_{0}>1$ and consequently by Kuang theorem, the endemic equilibrium point is asymptotically stable for $\tau \geq 0$. Therefore, both the Model-I and Model-II are asymptotically stable.

\subsection{Numerical simulation}

The numerical computation is done considering $b=0.88, p=0.8, \beta=0.15, d=0.03, \gamma=0.01, \tau=0$ then the diseases free equilibrium point is $(5.866,0,0)$ and the endemic equilibrium point is $(0.267$, $4.439,1.110)$ and for $\tau=5$ the endemic equilibrium point is $(0.310,3.791,0.948)$ and $R_{01}=22$, $\mathrm{R}_{0}=18.94, \mathrm{R}_{2}=18.59$. The graph of $\mathrm{S}$ and I are analyzed with considering the endemic equilibrium points.

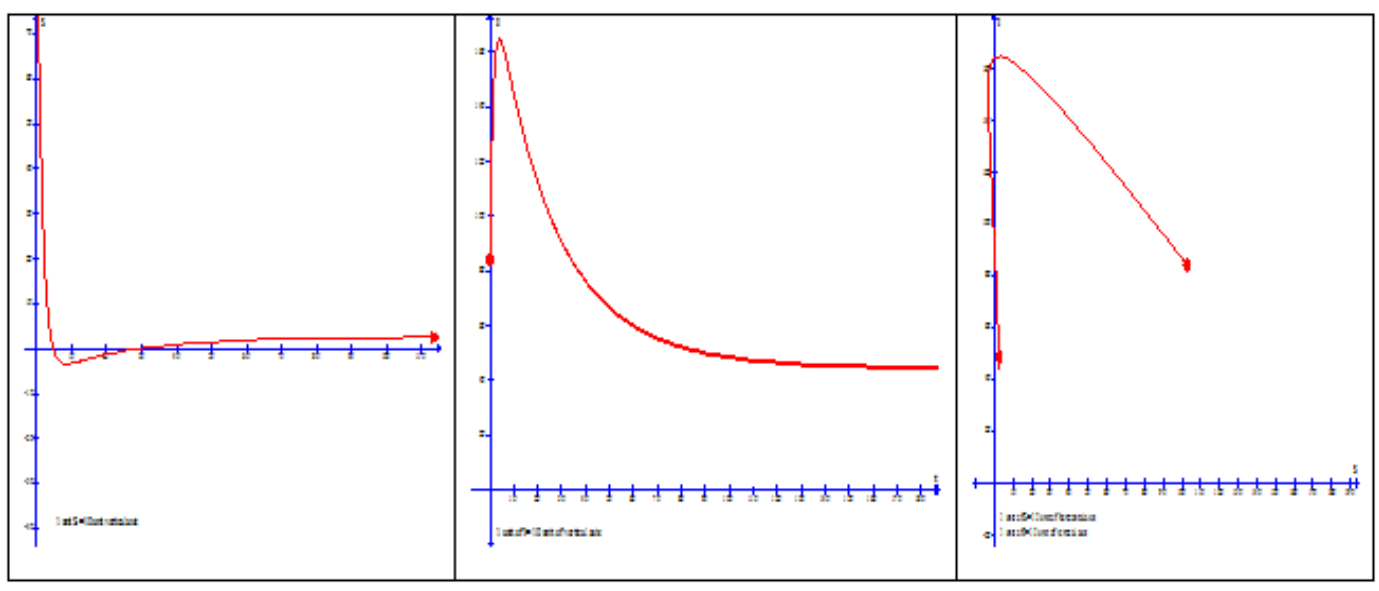

Fig-I: The graph of S-t, I-t and S-I for $\tau=0$. 


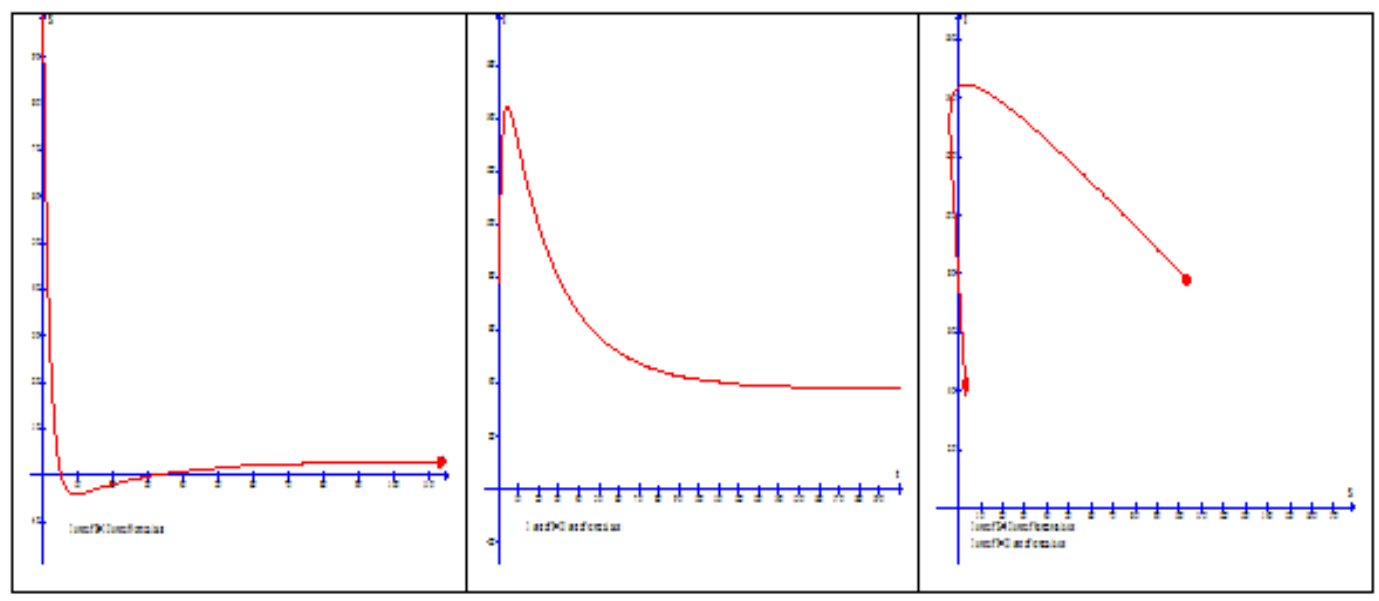

Fig-II: The graph of S-t, I-t and S-I for $\tau \neq 0$

From Figure-I and Figure-II it is clear that $\mathrm{S}$ and I both become asymptotically stable after long time and both cases after some time $S$ vanishes and becomes fixed after a long time.

\section{DISCUSSION}

From the above discussion it is clear that this SIR model in presence of vaccination to the newly entering individuals will be always stable. In delay (i.e. $\tau \neq 0$ ) considering the case the susceptible will decrease slowly compare to when $\tau=0$. For $\tau=0$ two conditions obtained for existence of endemic equilibrium points which are $R_{01}>1$, and for $\tau \neq 0$ other two conditions are obtained which are $R_{0}>1$ and $R_{2}>1$. It is clear from theoretical point and numerical simulation that the disease may be easily controlled in presence of vaccination to the newly entering individuals. Another important result is clear from the simulation that from $t=0$ to $t=t_{0}$ the total entry of susceptible (recovery and new entry ) is less than the total new infection and $t=t_{0}$ to $t_{1}$ the two numbers are same and after $t>t_{1}$ the first becomes greater compare to the second and ultimately it becomes asymptotic in nature.

\section{REFERENCE}

[1] A. Kaddar.(2010). Stability analysis of delayed SIR epidemic model with saturated incidence rate. Nonlinear Analysis. 3. 299-306.

[2] S. Ruang and Wang. W. (2003). Dynamical behavior of an epidemic model with a non-linear incidence rate. J. Differential equation. 188. 135-163.

[3] Arino, J., Mccluskey, C. C., and van den Driessche, P. (2003). Global results for an epidemic model with vaccination that exhibits backward bifurcation. SIAM J Appl Math 64: 260-276.

[4] Brauer, F. (2004). Backward bifurcations in simple vaccination models. J Math Anal Appl 298: 418431.

[5] Kribs-Zaleta, C. M. and Velasco-Hernadez, J. X. (2000). A simple vaccination model with mutiple endemic states. Math Biosci 164: 183-201.

[6] Liu, Wei-min, Hethcote, H. W., and Levin, S. A. (1987). Dynamical behavior of epidemiologic model with nonlinear incidence rates. J Math Biol. 25: 359-380. 
[7] Li, Jianquan, Ma, Zhien, and Zhou, Y (2006). Global analysis of SIS epidmic model with a simple vaccination and mutiple endemic equilibria. Acta Math Sci .26B: 83-93.

[8] Hui, Jing and Deming, Z. (2005). Global stability and periodicity on SIS epidemic models with backward bifurcation. Comp Math Appl 50: 1271-1290.

[9] Mena-Lorca, J. and Hethcote, H. W. (1992). Dynamic models of infectious diseases as regulators of population sizes. J Math Biol 30: 693-716.

[10] Li-Ming Cai and Xue-Zhi Li. 2009. Analysis of a SEIV epidemic model with non-linear incidence rate. Applied Mathematical Modelling. 33. 2919-2926.

[11] Ma. Z. and J. Li (eds.). 2009. Dynamical Modeling and Analysis of Epidemics. World Scientific.

[12] Hale, J. K. (1980). Ordinary Differential Equations. 2nd ed. Krieger, Basel.

\section{AUTHORS}

Dr. UTTAM GHOSH has obtained his M.Sc degree in Applied Mathematics from Calcutta University. He is also a Gold Medalist from the same university. He obtained his Ph.D degree from University of Kalyani in the year of 2013. He is now associated with Nabadwip Vidyasagar College, Nadia, West Bengal.

SOURAV CHOWDHURY obtained his M.Sc degree in Pure Mathematics from University of Klayani in the year of 2011. He is now associated with Global Institute of Management \& Technology, Krishnagar, Nadia, West Bengal.

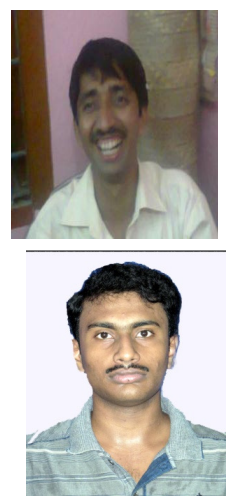

